\title{
Minimally invasive therapies in the treatment of bone malignancies
}

\section{Damian Dupuy}

Damian E. Dupuy M.D., "Minimally invasive therapies in the treatment of bone malignancies," Proc. SPIE 10297, Matching the Energy Source to the Clinical Need: A Critical Review, 1029708 (24 January 2000); doi: 10.1117/12.375228 


\title{
Minimally Invasive Therapies in the Treatment of Bone Malignancies
}

\author{
Damian E. Dupuy, M.D. \\ Brown University School of Medicine \\ Rhode Island Hospital \\ Providence, RI 02903
}

\begin{abstract}
In the past three decades many investigators have tried to apply thermally-mediated ablative procedures for the treatment of musculoskeletal neoplasms. Much of this preliminary work has been performed in animal models and small limited clinical studies. These studies have shown the techniques of cryosurgery, microwave, and radiofrequency (RF) to have some utility in specific clinical situations and specific tumors . To date the most widely utilized techniques are cryosurgery in the operative setting of malignant or locally recurrent neoplasms without cross-sectional image guidance and percutaneous radiofrequency ablation of osteoid osteomas with computed tomographic (CT) guidance. This paper will briefly review the various thermallymediated techniques. Since RF ablation is currently the only minimally invasive modality being applied to bone tumors the specifics of RF ablation in the treatment of benign and malignant tumors will be the primary focus of discussion.
\end{abstract}

Keywords: musculoskeletal neoplasms, therapy, radiofrequency ablation, cryosurgery, percutaneous

\section{Background and Significance}

In the past three decades many investigators have applied thermally-mediated ablative procedures for the treatment of musculoskeletal neoplasms. Much of this work has been performed in animal models and small limited clinical studies. These studies have shown the techniques of cryosurgery, and radiofrequency (RF) to have some utility in specific clinical situations and specific tumors. Experimentally and in the operating room microwaves have been used for providing local control of tumor, but to date no percutaneous application has been demonstrated. Laser energy has been used to cut bone in microsurgical techniques in the skull and head and neck region, but no clinical applications have been devised in the treatment of bone tumors. Focused ultrasound has not been specifically applied to bone tumors. To date the most widely utilized techniques are cryosurgery in the operative setting of malignant or locally recurrent neoplasms and percutaneous radiofrequency ablation of osteoid osteomas with computed tomographic (CT) guidance. RF ablation is the most robust percutaneous technique since it can provide a large region of coagulation necrosis with only a small entry wound.

This makes RF ablation a minimally invasive procedure that can be performed in an outpatient setting. This paper will briefly review the previously applied thermallymediated techniques and demonstrate the specifics of RF ablation in the treatment of benign and malignant bone and soft-tissue tumors.

Matching the Energy Source to the Clinical Need: A Critical Review, edited by Thomas P. Ryan, Proc of SPIE Vol 10297 (Vol. CR75), 1029708 - C) (2000) 2017 SPIE

CCC code: $0277-786 X / 17 / \$ 18 \cdot$ doi: $10.1117 / 12.375228$

161

Proc. of SPIE Vol. 10297 1029708-1 


\section{Cryosurgery}

The concept of tissue destruction by freezing has been in existence for the past century(1). Cryotherapy has been used to treat a wide variety of disorders including malignancy. In situ destruction was initially used to treat skin cancers. In the 1960's, development of a closed liquid nitrogen delivery system led to the expansion of uses for cryosurgery(2). The application of liquid nitrogen as a local adjuvant to curettage in the treatment of bone tumors was introduced three decades ago. Presently cryoablation is used to treat locally aggressive tumors in the bone (3), as well as brain, oropharynx, lung, breast, uterus, prostate, and liver(4). This technique has been shown to achieve excellent local control in a variety of benign-aggressive and malignant bone tumors. Early reports were associated with significant injuries to the adjacent bone and soft-tissue, resulting in high rates of fractures and infections. These early results reflected a lack of understanding of the potentially destructive effects of liquid nitrogen. More controlled applications and a better understanding of the destructive effects of liquid nitrogen have significantly reduced these early complications. To date only intraoperative techniques for bone tumor ablation have been performed since existing cryoprobe technology does not allow percutaneous use.

Liquid nitrogen stored below $-197^{\circ} \mathrm{C}$ can be used for both cryogenic preservation and tissue destruction. Tissue preservation can be performed by a slow freeze and a quick thaw whereas a quick freeze and slow thaw cause tissue destruction. Bone necrosis occurs when the temperature falls below $-21^{\circ} \mathrm{C}$. Destruction of tissue by freezing occurs by several mechanisms that are dependent on the rate of freezing. These include protein denaturation, osmotic driven shifts of intra and extracellular water, membrane destabilization, cellular rupture, and tissue ischemia. Fast cryoprobe cooling rates (500 ${ }^{0} \mathrm{C} / \mathrm{min}$.) produce different freezing patterns within the tissues than those produced by slower $(2$ to $300 \mathrm{C} \% \mathrm{~min}$.) cooling rates. Freezing rates are increased with close proximity to the cryoprobe. As freezing begins, ice crystals form within the vascular space as well as intracellularly, maintaining the structural architecture of the effected tissue. Intracellular ice begins forming when the cells are rapidly supercooled to -5 to $15^{\circ} \mathrm{C}$ in the presence of extracellular ice. Intracellular ice is lethal to cells. Cellular proteins denature and condense around ice crystals. Damage also occurs during a slow thaw phase as the intracellular ice crystals thaw and reform causing shearing disruption of organelles and cell membranes. Slower cooling rates are present at the periphery of the freeze zone. As the tissue temperature decreases, ice crystals form in the vascular space. Ice preferentially forms along small vascular channels since cell membranes pose a barrier to the propagation of ice. As the ice crystals form, solutes are concentrated in the surrounding fluid. This relative hyperosmolarity causes shift of intracellular water to the extracellular and vascular spaces. This leads to cellular dehydration, hyperosmolarity, and protein denaturation. The tissue architecture becomes grossly distorted as the vascular space expands up to 4 times its normal volume with contiguous ice crystals. Expansion to this size causes damage to the vascular endothelium and basement membrane. When thawing ensues, edema, vessel disruption and thrombosis lead to ischemic necrosis of the region. Also, as the ice crystals melt, there is a significant osmotic gradient between the extracellular and intracellular spaces. Since cell membranes have been destabilized, water rushes into the cells causing rapid cellular swelling and rupture. This process occurs during a slow cool phase at the periphery of the freeze ball. The middle region of the freeze zone is characterized by a variable intermediate rate of freezing. There can be any combination of intracellular and extracellular ice formation and cell dehydration. This freeze zone has the highest rate of cell survival. Tumors appear to freeze differently than normal tissue, which is very sensitive to freezing. It has been suggested that differences in tissue architecture, close cellular packing and less 
permeable cell membranes account for the altered freezing patterns. Tumor tissue is more resistant to cellular dehydration at slower cooling rates, thus expanding the intermediate freeze rate zone. Approximately $10 \%$ of cells remain viable following one freeze-thaw cycle.

Direct pouring of liquid nitrogen into a tumor cavity within bone or probe insertion into a small cavity are recognized techniques in the treatment of locally aggressive bone tumors. Both techniques require surgical curettage of the majority of tumor tissue and careful thermal isolation of the surrounding skin flaps, neurovascular bundle, and muscle to prevent normal tissue damage. Tumor spread into adjacent joints and soft tissue has to be prevented if local tumor eradication is to be successful. Direct pouring of liquid nitrogen into a cavity allows the liquid nitrogen to come in contact with any residual viable tumor lining the wall. These tumor cavities are often irregular and cryoprobes can not adequately approximate the walls unless the tumor cavity is small as in the small bones of the hands and feet. If the cavity is very regular and smooth in geometry gel coupling materials can be placed in the cavity and cooled with a centrally placed cryoprobe. These techniques can destroy tumor lining the cavity up to $2 \mathrm{~cm}$ in thickness(5). Cryosurgery does not have a role for high grade malignant neoplasms since these tumors tend to have extension of tumor cells into adjacent soft tissues which can be more infiltrative and less defined. Since long term patient survival as well as limb preservation are the primary objectives cryosurgical techniques have not been successful in these patients (3). However, in locally recurrent neoplasms that rarely spread to other sites, cryosurgery provides additional tumor kill without more extensive local surgery(6). Recently, Malawer et al have shown in 102 patients with giant cell tumor who were followed for a minimum of four years that a high cure rate can be achieved with a low complication rate(7). These patients had a less than $6.0 \%$ incidence of pathologic fracture. This data shows that cryosurgery is an excellent adjunct for the treatment of this locally aggressive tumor. Cryosurgery enables joint preservation, excellent functional outcome, and low recurrence rates when compared with other joint preservation procedures. Current cryosurgical probes have not been applied to bone cancer therapy with percutaneous minimally invasive techniques. For future development smaller probes with the ability to treat focused areas without harming adjacent tissues are necessary if this technique is to be applied bone tumors percutaneously.

\section{Radiofrequency Ablation}

Radiofrequency (RF) ablation was pioneered in 1920 by Harvey Cushing for the creation of small lesions within the central nervous system. Since that time the technique has been refined such that precise control of lesion size can be achieved by measuring the local temperature and electrical resistance within the tissues being treated. Ablating neural tissue with RF has been successfully used for the treatment of pain from trigeminal neuralgia (8), facet osteoarthritis and failed back syndrome (9). RF ablation's ability to create localized necrotic lesions is also why it is well established as the treatment of choice for many symptomatic cardiac arrhythmias (10) and small painful benign bone tumors called osteoid osteomas (11). The clinical efficacy of RF ablation in these areas has been clearly established. Although the application of cryosurgical techniques to musculoskeletal tumors has been around for over threes decade, imageguided ablative therapies using thermal energy sources such as $R F$ have received recent attention as minimally invasive strategies to treat musculoskeletal tumors .

$\mathrm{RF}$ ablation is a technique whereby an alternating electrical current operating in the frequency of radiowaves $(460-480 \mathrm{kHz})$ is emitted from the tip of an electrode or needle placed directly into tissues. 


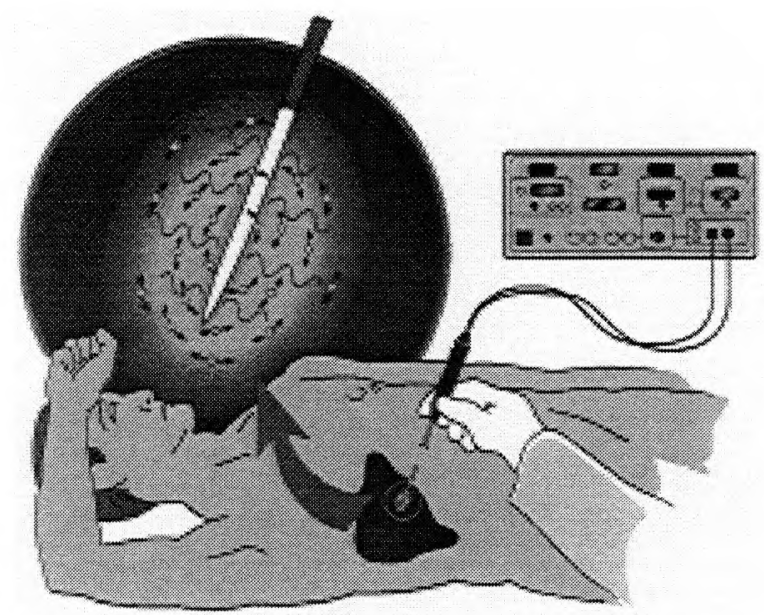

Figure 1

Diagram of hepatic RF ablation illustrating the percutaneous technique.

The alternating current causes the local ions to vibrate producing heat and inducing cell death by coagulative necrosis. The cytotoxic temperature threshold is $50^{\circ} \mathrm{C}$., but with RF ablation techniques temperatures can exceed this and actually reach the boiling point of water. Until recently, a major limitation of RFA was the small lesion size created by this technique. Within the past few years technical advances in RF systems have improved such that heat lesions larger than $5 \mathrm{~cm}$ in diameter can be created with a single treatment (12).

There are currently three RF systems that are being utilized for treating tumors. Two of the systems (RITA Medical Systems Inc., Mountain View, CA- 50 watt output, electrodes measure temperature and impedance and Radiotherapeutics- Boston Scientific, Natick, MA- 90 Watt output, electrodes measure impedance only) utilize a "jack-hook" deployable array RF electrode which consists of four to sixteen small wires which are deployed through a 15 -gauge needle.

\section{Figure 2}

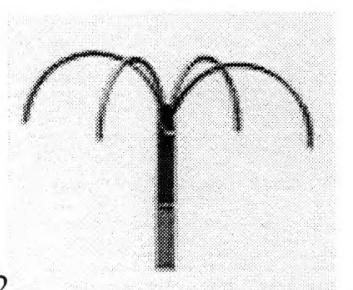

Image of the RITA RF electrode showing four deployable needles extending beyond the 15 gauge introducer needle.

The "jack-hook" needle has not been applied to bone lesions since deployment of the thin wires could be difficult if the cancellous and cortical bone were still intact. The third RF system (Radionics Inc., Burlington, MA, 200 watt output, electrodes measure 
temperature and impedance) utilizes either single non-perfused or single and triple "cluster" perfused electrodes.

Figure 3

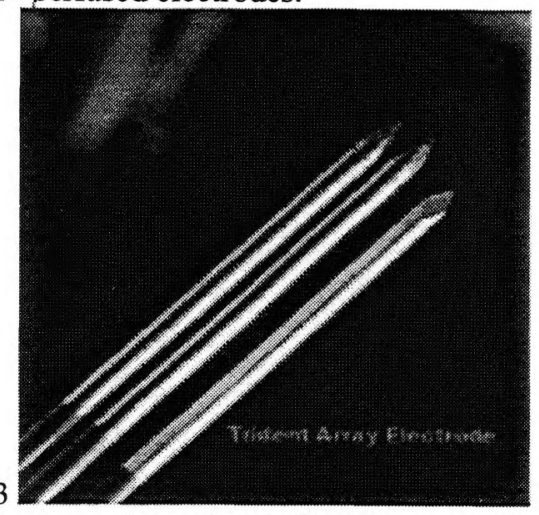

Close-up view of the cluster Cool-tip electrode (Radionics,Inc,Burlington,MA) which consists of three 17 gauge needles each with a $2.5 \mathrm{~cm}$ active tip which has been reported to achieve thermocoagulation diameters as large as $7 \mathrm{~cm}$ in cirrhotic liver patients.

The non-perfused conventional thermistor electrodes are of approximately 18 gauge and have a small $5 \mathrm{~mm}$ exposed tip. These smaller electrodes can ablate tissue with a treatment diameter of approximately $10-16 \mathrm{~mm}$ and are used for the ablation of osteoid osteomas or small tumors. The internally cooled RF electrode can increase the volume of induced coagulation necrosis to 4 to 7 centimeters as reported in liver (13), but may be greater in soft tissue malignancies due to the diminished cooling effect of the regional blood flow. Perfusion of the RF electrode tip reduces the local impedance thus allowing a greater radius of RF energy deposition .

Figure 4

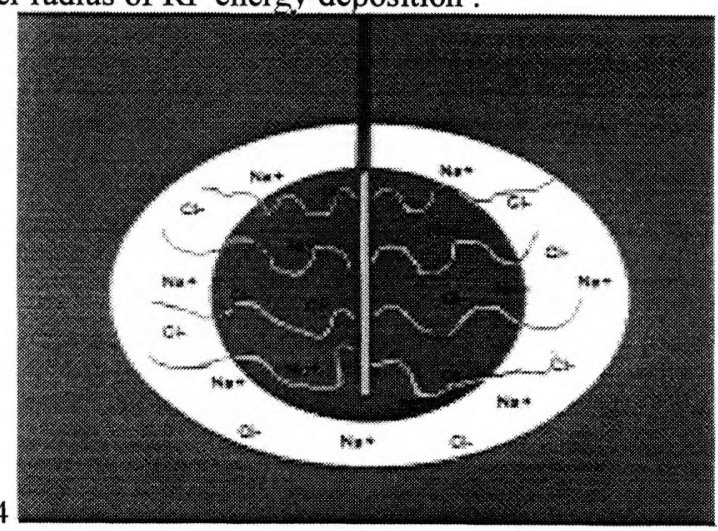

Schematic illustrating the greater diameter of thermocoagulation obtained with the Cool-tip $\mathrm{RF}$ electrode (Radionics,Inc,Burlington,MA). Lighter peripheral zone represents greater area of thermocoagulation (tip-cooling) compared with central darker zone (no tip cooling).

The perfused treatment probes have a diameter of 18 gauge, but a thin electrically insulated material coating increases its effective diameter to approximately 17 gauge. The treatment probe has 2 to $3 \mathrm{~cm}$. of an uninsulated tip. Each probe contains a radiofrequency electrode with a thermocouple embedded at the tip of the probe to measure temperature. Circuitry incorporated within the generator and probe permits continuous measurement of tissue impedance. Two internal lumens extend to the probe tip. One is used to deliver chilled perfusion fluid (sterile saline or distilled water) to the probe tip, while the second returns the perfusion effluent to a collection unit located external to the 
patient. Thus the system is entirely closed, so that no perfusion fluid from within the treatment probe comes into contact with the patient. Tubing from the standard infusion pump is connected to the perfusion inlet port of each treatment probe. Additional tubing is connected to the perfusion outlet port and leads to a collection unit. Prior to the procedure and appropriate amount of perfusion fluid is appropriately chilled so that ice of slush is visible within the bag at the time treatment is initiated. A standard peristaltic pump is used to infuse ice-cold $\left(\sim 0^{\circ} \mathrm{C}\right)$ perfusion fluid into the cooling lumen of the treatment probe . A grounding pad or pads is/are placed on the patient's thigh(s). If a single RF treatment probe is used, at least two grounding pads $\left(96 \mathrm{~cm}^{2}\right.$ surface area each) or the equivalent (minimum total surface area of approximately $200 \mathrm{~cm}^{2}$ ) must be used. In the event that a 3-probe cluster is used, at least 4 grounding pads, or their equivalent (approximately $400 \mathrm{~cm} 2$ surface area) must be used. The pads must be placed horizontally to maximize the grounding effect thus preventing thermal injury to the skin. The grounding pad(s) and treatment probe(s) are connected to the radiofrequency generator. When the RF generator is activated, current flows between the conductive electrode tip and the grounding pad(s) or "dispersive electrode". The increase in the tissue temperature is proportional to the current density. Since the current density is highest near the conductive electrode tip, coagulation is induced in the tissue surrounding the treatment probe. When performing a RF treatment the coagulation depth is controlled from the length of the uninsulated "active" electrode tip. The diameter of coagulation necrosis produced around the tip of the treatment electrode depends on the current, duration of treatment and local tissue blood flow. Based on our in vivo experience using perfusion electrodes and pulsed current technique in liver tumors with concomitant cirrhosis and soft tissue tumors, areas of the tissue up to $4.2 \mathrm{~cm}$. in diameter may be induced with a single electrode and up to $7.3 \mathrm{~cm}$. in diameter with a cluster electrode.

RF has been applied to both benign and malignant bone tumors. The first use was for the thermocoagulation of small painful benign bone tumors called osteoid osteomas (15). Osteoid osteomas predominantly occur in the pediatric age group and arise within the cortex of long bones. The cause of these tumors is unknown. Nocturnal pain relieved with anti-inflammatory drugs is the classic clinical scenario exhibited by these patients. Pain is related to prostaglandin production within the cells of the small, central, vascularized tumor nidus. Most of these tumors require removal either by surgical or percutaneous methods. Occasionally, these tumors spontaneously regress. Surgical removal is the most expensive treatment and requires a cortical osteotomy with the accompanying risks of anesthesia, surgical wound complication and incomplete removal (11). RF ablation can be performed under intravenous sedation or general anesthesia using CT guidance. Patients typically leave the hospital several hours after the procedure. The lesion is initially localized with CT and a safe percutaneous route is planned to avoid vital neurovascular structures. A core of bone is then removed with a bone-cutting biopsy needle. Once the overlying dense cortical bone has been traversed to the center of the osteoid osteoma a conventional (non-internally cooled) RF electrode is placed within the center. The internally-cooled RF electrodes are not needed in this application because the area of treatment is almost always under $10 \mathrm{~mm}$ in diameter. A single grounding pad is placed on the skin of the opposing surface from the electrode placement. CT confirms satisfactory positioning and the RF generator connected to the electrode is activated. 

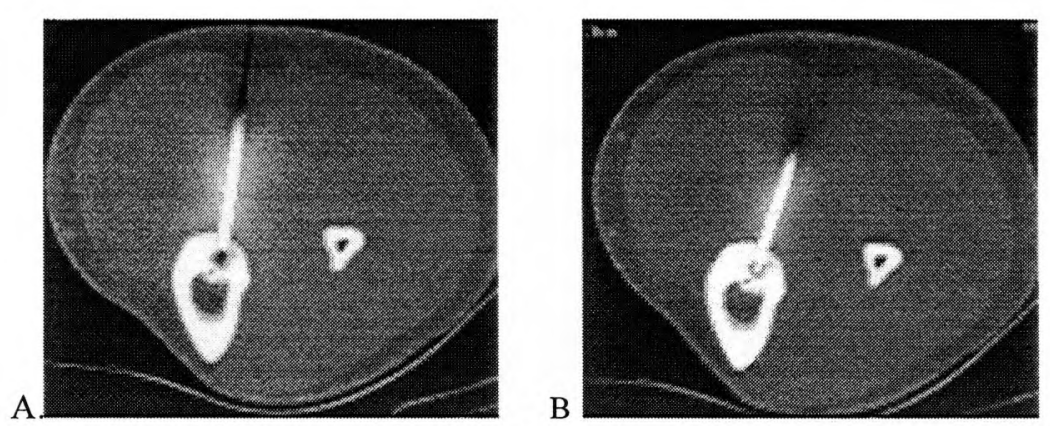

Figure 5 Axial CT image of the tibia in a 21 year old man with night pain and shows a typical osteid osteoma. Note the bone biopsy needle positioned directly above the nidus (A). The core of bone is removed and a small conventional RF electrode is placed directly into the nidus (B)

Axial CT image with the patient prone showing the RF electrode within the nidus of the osteoid osteoma

A 6 minute RF energy application with the temperature maintained at $90{ }^{\circ} \mathrm{C}$. is almost always sufficient to destroy the small central nidus of prostaglandin producing cells. The ouput in watts is typically less than 10 and the impedance is usually less than 150 ohms. The electrode is removed and a small bandage is applied to the skin. Patients typically have one to two days of procedure pain different than the pain from the osteoid osteoma. Relief of the osteoid osteoma pain almost always occurs within the first 24-48 hours. Cure with one session can be as high as $90 \%(11)$ and retreatment can be performed if the first treatment is not immediately successful or the patient's pain returns in the future. Follow-up imaging is usually unnecessary since the small ablated area does not cause significant bone weakening. The ablated region of bone typically undergoes dimineralization after about 6 weeks. Healing of the thermocoagulation defect is slow and radiographic changes may take as long as one year. No significant complications have been reported in the literature.

Preliminary studies in malignant bone tumor treatment with RF is showing promise. Previously irradiated foci of tumor either primary or metastatic which are still biologically active can be treated locally with internally-cooled RF electrodes (14). Pain reduction, control of hemorrhage and local tumor irradication can be performed in both the axial and appendicular skeleton.

Figure 6

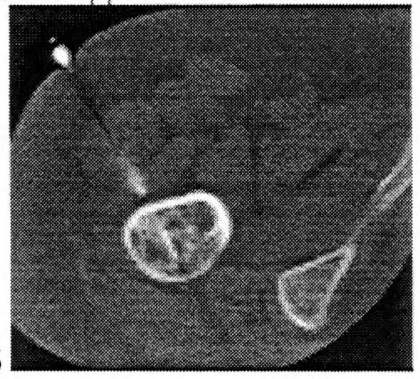

Axial CT image of the proximal right femur in a 69 year old woman with metastatic lung cancer. The patient had persistent pain despite a complete course of external beam radiotherapy. A $3 \mathrm{~cm}$ active tip internally-cooled RF electrode was placed into the metastatic deposit. Significant pain relief was achieved.

In areas where tumor abuts vital structures such as the spinal cord RF may not be effective since local thermal injury may not be desirable. Spinal RF can be performed in 
the vertebral body when the cortex between the electrode and the spinal canal is intact (16).

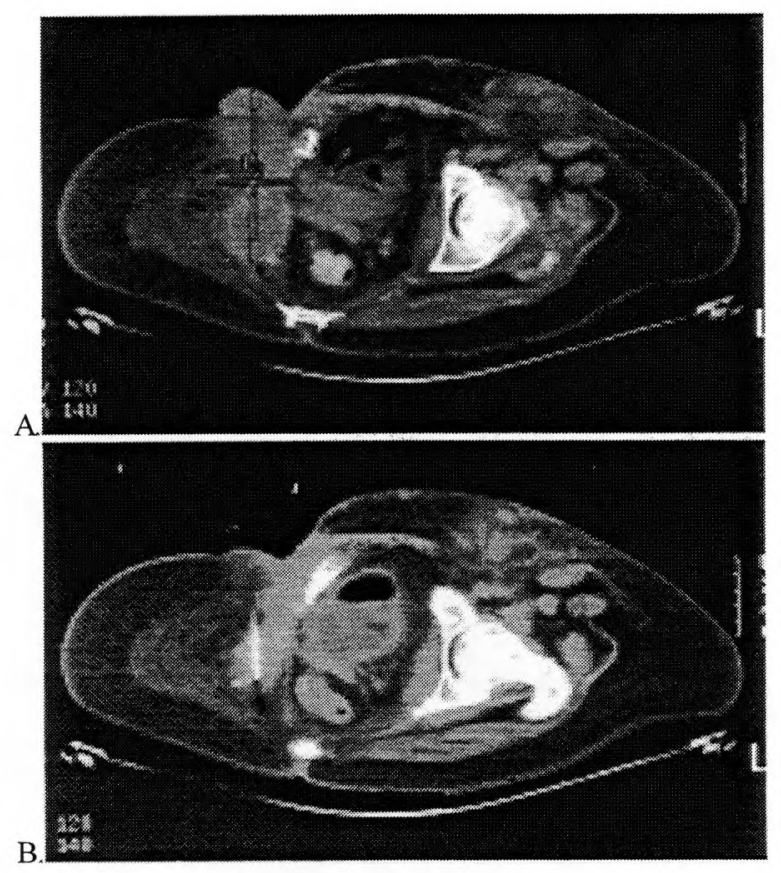

Figure 6. Axial CT images in a 61 year old woman s/p right hemipelvectomy for malignant fibrous histiocytoma. The patient had recurrent tumor which was growing through a surgical wound causing extensive bleeding. An initial planning image (A) measures the tumor geometry and distance between the tumor and patients rectum. A single $3 \mathrm{~cm}$ active tip RF electrode was placed into the deep tumor margin (B). The patient's bleeding was controlled and the majority of the tumor mass underwent necrosis.

The temperature changes within the spinal canal during vertebral body RF application in this study went from $37^{\circ} \mathrm{C}$. to a maximum mean of $48^{\circ} \mathrm{C}$., $41^{\circ} \mathrm{C}$., and $39^{\circ} \mathrm{C}$. at a distant from the RF electrode of 5,10 and $15 \mathrm{~mm}$, respectively over a $15 \mathrm{~m}$ period compared to a maximum of $84^{\circ} \mathrm{C}$., $62^{\circ} \mathrm{C}$., and $58^{\circ} \mathrm{C}$. in paraspinal muscle. Cortical and cancellous bone are relative insulators of RF energy compared with soft tissue. The bone and pulsating cerebrospinal fluid between the spinal canal and vertebral body may act as heat sinks dispersing the RF energy. 
A.

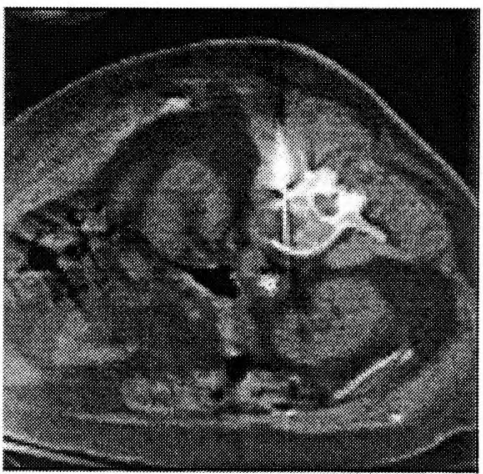

B.

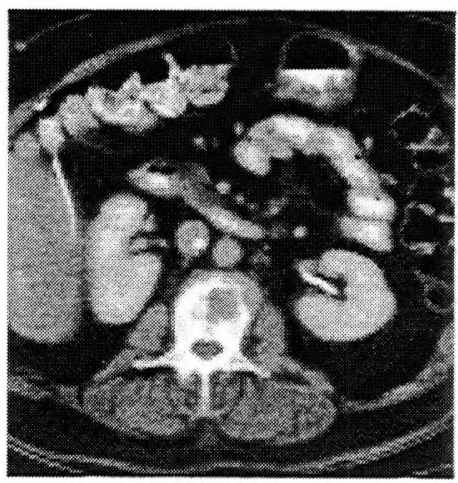

Figure 7. Axial CT images in a 55 year old woman with metastatic hemangiopericytoma. The tumor involved the second lumbar vertebral body, but an intact posterior cortex was identified. Thermocoagulation of the tumor was achieved with a single RF treatment (A). A follow-up CT three months later shows posterior vertebral body sclerosis, but no extension of tumor into the spinal canal. The RF therapy was successful at preventing future spinal canal encroachment by tumor.

This has been shown in an animal model and in vitro experiments. ( DE Dupuy and SN Goldberg personal communication).

Figure 8: Comparison of tissue heating using radiofrequency ablation techniques

\begin{tabular}{|c|c|c|c|c|c|}
\hline & $\underline{N}$ & $\frac{\text { Mean Current }}{(\mathrm{mA})}$ & $\frac{\text { Tip temperature }}{\frac{\text { Range }}{\left({ }^{\circ} \mathrm{C}\right)}}$ & $\frac{\text { Temp Increase }}{\text { at } 10 \mathrm{~mm}\left({ }^{\circ} \mathrm{C}\right)}$ & $\underline{\text { Significance }}$ \\
\hline \multicolumn{6}{|l|}{ In-vivo: } \\
\hline vertebra & 5 & $170 \pm 35$ & $20-25$ & $5 \pm 1$ & $\mathrm{P}<0.001$ \\
\hline muscle & 5 & $326 \pm 42$ & $20-25$ & $31 \pm 3$ & \\
\hline \multicolumn{6}{|l|}{ Ex-vivo: } \\
\hline $\begin{array}{l}\text { Cancellou } \\
\text { s bone }\end{array}$ & 5 & $139 \pm 29$ & $95 \pm 1$ & $13.4 \pm 4.5$ & $\mathrm{P}<0.01$ \\
\hline $\begin{array}{l}\text { Cortical } \\
\text { bone }\end{array}$ & 11 & $455 \pm 92$ & $94 \pm 1$ & $11.2 \pm 2.0$ & $\mathrm{P}<0.01$ \\
\hline Liver & 6 & $194 \pm 21$ & $95 \pm 2$ & $20.0 \pm 3.4$ & \\
\hline Agar & 5 & $586 \pm 63$ & $94 \pm 3$ & $18.5 \pm 3.1$ & \\
\hline
\end{tabular}

N.B. RF applied for 10 minutes in-vivo and 6 minutes ex-vivo. For in-vivo studies, tip temperatures were maintained at $20-25^{\circ} \mathrm{C}$ by internal electrode cooling and maximum current without impedance rise was administered. For ex-vivo studies, RF was applied without cooling at tip temperature of $94 \pm 2^{\circ} \mathrm{C}$. Lower temperatures were generated in both types of bone compared to liver or agar phantoms.

The treatment of bone tumors with direct cytotoxic therapy with a minimally invasive CT-guided procedure may provide a cost effective means for local tumor control. In certain patients RF alone may provide a means of tumor irradication. In larger tumors a combination of RF and external beam radiotherapy may improve local recurrence rates. In theory, thermocoagulation of the central, less vascular tumor (often not effectively treated with radiation) with RF may make the peripheral, well oxygenated tumor more effectively treated with radiation. This is currently being evaluated at several centers. 
Regarding RF and its ability to treat large areas of tumor within bone, the technology is present, but the clinical applications are still being defined. Large multi-center trials are being devised to evaluate the efficacy at pain control in sites of metastatic disease. In primary malignancies of bone minimally invasive strategies are far away from clinical implementation since the majority of patients undergo standard surgical removal and reconstruction. In non-surgical candidates RF may provide a minimally invasive alternative for local tumor irradication.

\section{Microwave}

Microwave like RF can provide direct thermocoagulation of tumor. Percutaneous microwave therapy for hepatic tumors has been performed(17). In direct comparison with $\mathrm{RF}$, smaller areas of thermocoagulation are achieved. To date the percutaneous approach has not been applied to bone lesions. Fan et al (18) treated 62 patients with various bone tumors with direct microwave-induced hyperthermia in the operative setting. The surgical procedure consisted of isolating the tumor from adjacent normal structures . During tumor heating with a microwave antenna array the normal tissues were cooled to below $39^{\circ} \mathrm{C}$. with a water circulation system. Central tumor temperatures reached the boiling point whereas the surface temperatures were still well above cytotoxic ranges. Treatment duration lasted approximately 50 minutes. Local tumor control was acheived in 57/62 patients with five patients having local fracture complications. Like cryosurgery this approach requires operative isolation of the tumor to prevent necrosis of surrounding normal tissues. Future work with microwave technology is necessary to allow the treatment of larger tumor volumes percutaneously. Advantages of microwave over RF are the shorter treatment times which have been shown in liver. Disadvantages as mentioned before are the size of thermocoagulation which to date has not been fully evaluated in bone in animals or humans.

\section{Conclusions}

Many types of energy can be used to destroy tumor. In bone neoplasms RF, cryosurgery and microwaves have been tried. To date only RF has been used as a minimally invasive percutaneous treatment. The technology is young and aside from the small benign osteoid osteomas which have been treated with RF for over 7 years the treatment of malignancies is in the preliminary stages of assessment. The safety and efficacy in the treatment of larger tumor volumes is ongoing. Multicenter trials are necessary and are currently in the planning stages.

\section{References:}

1. Gage AA. History of Cryosurgery. Sem Surg Onc 1998; 14, 99-109.

2. Cooper IS. Cryogenic surgery of the basal ganglia. JAMA 1962;181:600-604

3. Bickels J, Meller I, Shmookler BM, Malawer MM. The role and biology of cryosurgery in the treatment of bone tumors. A review. Acta Orthop Scand 1999;70:308-15

4. Bayjoo P, Jacob G. Hepatic cryosurgery: Biological and clinical considerations. J Royal Coll Surg Edinb 1992; 37: 369-372.

5. Marcove RC, Weis LD, Vaghaiwalla MR. Cryosurgery in the treatment of giant cell tumor of bone. A report of 52 consecutive cases. Cancer 1978;41:957-969. 
6. Malawer MM, Dunham W. Cryosurgery and acrylic cementation as surgical adjuncts in the treatment of aggressive (benign) bone tumors. Analysis of 25 patients below the age of 21. Clin Orthop $1991262: 42-57$

7. Malawer MM, Bickels J, Meller I, Buch RG, Henshaw RM, Kollender Y.Cryosurgery in the treatment of giant cell tumor. A long-term followup study. Clin Orthop 1999;359:176-88

8. Sweet WH, Wepsic JG. Controlled thermocoagulation of trigeminal ganglion and rootlets for differential destruction of pain fibers. $J$ of Neurosurg. 1974;40:143156.10-17

9. Cho J, Park YG, Chung SS Percutaneous radiofrequency lumbar facet rhizotomy in mechanical low back pain syndrome. Stereotact Funct Neurosurg 1997;68:212-7

10. De Giovanni JV. Treatment of arrhythmias by radiofrequency ablation. Archives of Disease in Childhood. 1995;73(5):385-7

11. Rosenthal DI, Hornicek FJ, Wolfe MW, Jennings LC, Gephart MC, and Mankin HJ. Changes in the management of osteoid osteoma. J Bone Joint Surg 1998; 80:815-821

12. Goldberg SN, Gazelle GS, Solbiati L, Rittman Wj, Mueller PR. Radiofrequency tissue ablation: increased lesion diameter with a perfusion electrode. Acad Radiol 1996;3:636-644.

13. Goldberg SN, Solbiati, Hahn PF, Cosman E, Conrad JE, Fogle R, Gazelle GS. Large-volume tissue ablation with radio frequency by using a clustered, internally cooled electrode technique: laboratory and clinical experience in liver metastases. Radiology 1998: 209:371-379.

14. Dupuy DE, Safran H, Mayo-Smith WW, Goldberg SN Radiofrequency ablation of painful osseous metastatic disease. Radiology 1998;209(P):389.

15. Rosenthal DI, Springfield DS, Gebhart MC, Rosenberg AE, and Mankin HJ Osteoid osteoma: percutaneous radiofrequency ablation. Radiology 1995;197:451454.

16. Dupuy DE, Goldberg SN, Gazelle GS, and Rosenthal DI. Cooled-tip radiofrequency ablation in the vertebral body: temperature distrubution in the spinal canal. Radiology 1997;205(P);330.

17. Murakami R, Yoshimatsu S, Yamashita Y, Matsukawa T, Takahashi M and Sagara $\mathrm{K}$. Treatment of hepatocellular carcinoma: Value of percutaneous microwave coagulation. AJR 1995; 164:1159-1164

18. Fan QY, Ma BA, Qlu XC, Li YL, Ye J, Zhou Y. Preliminary report on treatment of bone tumors with microwave-induced hyperthermia. Bioelectromagnetics 1996;17:218-22

Correspondence: Email: ddupuy@lifespan.org;

WWW; http://www.brown.edu/Departments/Diagnostic_Imaging/acad/sota.html\#rf;

Telephone:401-444-5184; Fax: 401-444-5017 